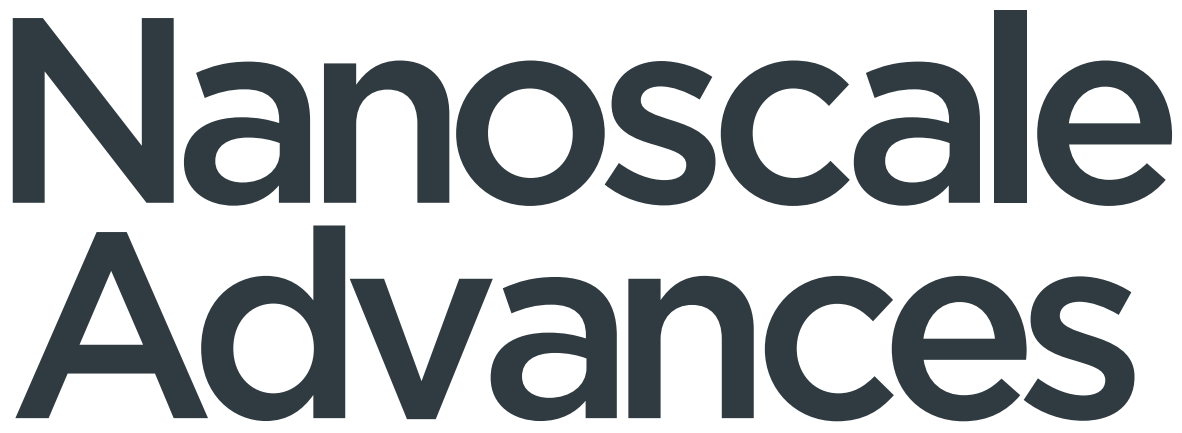

Volume 2

Number 6

June 2020

Pages 2205-2612 


\title{
Nanoscale Advances
}

Check for updates

Cite this: Nanoscale Adv., 2020, 2, 2326

Received 16th February 2020

Accepted 4th May 2020

DOI: 10.1039/d0na00130a

rsc.li/nanoscale-advances

\section{Bioinspired selective synthesis of liquid-crystalline nanocomposites: formation of calcium carbonate- based composite nanodisks and nanorods $\uparrow$}

\author{
Masanari Nakayama, (iD) a Satoshi Kajiyama, (D) a Akihito Kumamoto, (DD ${ }^{\mathrm{b}}$ \\ Yuichi Ikuhara iD ${ }^{b}$ and Takashi Kato iD *a
}

\begin{abstract}
Here we report new organic/inorganic hybrid colloidal liquid crystals that consist of colloidal calcium carbonate $\left(\mathrm{CaCO}_{3}\right) /$ poly(acrylic acid) (PAA) hybrid nanodisks. We selectively synthesized anisotropic liquid-crystalline $\mathrm{CaCO}_{3}$-based nanodisk and nanorod composites in water/methanol mixtures, which formed discotic and calamitic nematic liquid crystals in their colloidal dispersions, respectively. The vaterite nanodisks and calcite nanorods were selectively synthesized in methanol-rich and water-rich solutions, respectively. The observation of these materials with transmission electron microscopy clarified the atomic-scale structures of these nanodisks and nanorods, revealing the self-organized $\mathrm{CaCO}_{3} /$ PAA hybrid structures with the ability to form colloidal liquid crystals. The liquid crystals were prepared under mild and aqueous conditions by methods using acidic polymers inspired by the biomineralization process. The present approach provides new insights into the design of organic/ inorganic hybrid colloidal liquid crystals and development of environmentally friendly functional hybrid materials.
\end{abstract}

\section{Introduction}

Liquid crystals are dynamic ordered molecular assemblies. ${ }^{\mathbf{1 , 2}}$ They have been intensively studied as functional materials for a broad range of applications in fields including energy, information, environment, and medicine. Normal liquid crystals consist of molecular components. Recently, a variety of colloidal liquid crystals have been developed ${ }^{3,4}$ that are composed of materials such as biomacromolecules, ${ }^{5-7}$ viruses, ${ }^{8-10}$ minerals, ${ }^{11-13}$ and organic/inorganic hybrids. ${ }^{14}$ For example, hydroxyapatite/polymer hybrid nanorods exhibit colloidal liquid-crystalline (LC) states that are responsive to magnetic fields and biocompatible properties..$^{15-17}$

Development of sustainable materials is important to address global environmental issues. Biominerals are structurally controlled biological hard tissues such as nacre from seashells and egg shells, which are good models for sustainable materials. ${ }^{18-22}$ These biominerals are composed of biopolymers and $\mathrm{CaCO}_{3}$ and formed by energy-saving processes that involve self-organization of inorganic and organic components to form

\footnotetext{
${ }^{a}$ Department of Chemistry and Biotechnology, School of Engineering, The University of Tokyo, 7-3-1 Hongo, Bunkyo-ku, Tokyo 113-8656, Japan. E-mail: kato@chiral.t. u-tokyo.ac.jp

${ }^{b}$ Institute of Engineering Innovation, School of Engineering, The University of Tokyo, 211-16 Yayoi, Bunkyo-ku, Tokyo 113-8656, Japan

† Electronic supplementary information (ESI) available: Supplementary figures (Fig. S1-S13). See DOI: 10.1039/d0na00130a
}

intricate nanostructures. ${ }^{23-25}$ ACC precursors have been formed in the presence of biopolymers ${ }^{26-28}$ and then the crystallization has been precisely controlled to form uniform nanocrystals with well-tuned crystal morphologies, polymorphs, sizes, and orientations under mild conditions. ${ }^{29-33}$ For example, in seashells, uniform rod-shaped calcite crystals form in the prismatic layer, ${ }^{34}$ whereas disk-shaped aragonite crystals form in the nacreous layer. ${ }^{35}$ Although crystallization of ACC is yet to be fully understood, it has been proposed that ACC can crystallize through different pathways depending on the conditions. ${ }^{36-40}$

Anisotropic $\mathrm{CaCO}_{3}$ particles with plate-like or ellipsoid-like morphologies were synthesized by simple solution-based methods of controlling concentrations of salts and molecular additives, mixed solvent compositions, $\mathrm{pH}$ values, temperature, and mixing speed and time of the reaction solutions. ${ }^{\mathbf{4 1 - 4 4}}$ However, it is still challenging to obtain $\mathrm{LC} \mathrm{CaCO}_{3}$-based particles having large aspect ratios and high colloidal stability. Our intention is to develop new LC nanocomposites by applying the bioinspired crystallization control using ACC precursors to the formation of anisotropic colloidal mesogens. In our previous study, calamitic $\mathrm{CaCO}_{3}$ liquid crystals consisting of calcite nanorods were synthesized from amorphous $\mathrm{CaCO}_{3}$ (ACC) precursors stabilized with poly(acrylic acid) (PAA). ${ }^{\mathbf{1 4}}$

In this paper, we report the first synthesis of discotic $\mathrm{CaCO}_{3}$ liquid crystals consisting of vaterite nanodisks by tuning the crystallization conditions for the ACC precursors. The vaterite nanodisks and calcite nanorods were selectively synthesized by tuning methanol concentration of colloidal dispersions of ACC 
precursors. Transmission electron microscopy (TEM) analyses of the nanodisk and nanorod composites are also described.

\section{Results and discussion}

We synthesized $\mathrm{LC} \mathrm{CaCO}_{3}$-based composite nanodisks and selectively synthesized discotic liquid crystals consisting of $\mathrm{CaCO}_{3}$-based nanodisks and calamitic liquid crystals consisting of $\mathrm{CaCO}_{3}$-based nanorods through crystallization control of ACC precursors by changing the composition of the crystallization solution (Fig. 1 and 2).

ACC precursors were dispersed in $\mathrm{Na}_{2} \mathrm{CO}_{3}$ solution to induce their crystallization (Fig. 1). Poly(acrylic acid) (PAA) was used as a macromolecular additive to stabilize ACC. ${ }^{45}$ Crystallization of the ACC precursors was tuned by changing the solvents. We found that $\mathrm{CaCO}_{3}$-based composite nanodisks selectively formed in methanol/water $(7: 3)$ solution (Step 1A in Fig. 1), whereas $\mathrm{CaCO}_{3}$-based composite nanorods selectively formed in water (Step 1B in Fig. 1). Concentrated colloidal dispersions of these colloidal nanoparticles exhibited LC states (Step 2A and 2B in Fig. 1). TEM observations of the nanodisk crystals showed that they selectively formed after crystallization of the precursors in $25 \mathrm{mM} \mathrm{Na}_{2} \mathrm{CO}_{3}$ methanol/water $(7: 3)$ solution (Fig. 2a). Disk-like crystals were also observed by scanning electron microscopy (SEM) (Fig. S1 $\dagger$ ). The average diameter of the nanodisks estimated from TEM images was $320 \pm 74 \mathrm{~nm}$. The thickness of the nanodisks was $137 \pm 80 \mathrm{~nm}$, which was estimated by atomic force microscopy (AFM) observation (Fig. 2b, c, and $\mathrm{S} 2 \dagger$ ). The aspect ratio of the nanodisks was around 2.5. When the crystallization solution was changed from methanol/ water $(7: 3)$ to pure water, the same ACC nanoparticle precursors transformed into nanorods (Fig. S3a-c $\dagger$ ). The nanorods were $419 \pm 89 \mathrm{~nm}$ long and $126 \pm 25 \mathrm{~nm}$ wide, giving an aspect ratio of around $3.3 .{ }^{14}$ The shape anisotropy of the nanodisks induced LC properties in concentrated colloidal dispersions. Aqueous colloidal dispersions of nanodisks with various concentrations were observed by polarizing optical microscopy (POM). When the volume fraction of nanodisks was $1.7 \mathrm{vol} \%$, a dark image was observed (Fig. 2d), indicating that the nanodisks formed an isotropic state in the colloidal dispersion. At a concentration of $16 \mathrm{vol} \%$, the dispersions partly showed fluidic birefringence (Fig. 2e). When the dispersion concentration was raised to $22 \mathrm{vol} \%$, LC texture was observed over the whole region under crossed polarizers (Fig. 2f). These observations suggest that the nanodisks self-assembled into LC states at concentrations above $16 \mathrm{vol} \%$. Concentrated colloidal dispersions of the composite nanorods also showed LC states caused by their shape anisotropy (Fig. S3d $\dagger$ ).$^{14}$ Interestingly, colloidal stability was observed for the nanodisks and nanorods without surface modification (Fig. S4 $\uparrow$ ). The zeta potentials measured for the nanodisks and nanorods were $-17.4 \pm 6$ and $-14.7 \pm 6 \mathrm{mV}$, respectively (Fig. S5 $\dagger$ ). Therefore, the surfaces of these materials were negatively charged. It is considered that PAA covered the nanoparticle surfaces to provide colloidal stability, which is important for the formation of colloidal LC states. ${ }^{15}$

To obtain further insight into the LC properties of the nanodisks and nanorods, we analyzed their structures using conventional TEM, aberration-corrected high-resolution TEM (HRTEM), and cryogenic HRTEM (cryo-HRTEM) (Fig. 3). The polycrystalline structures of the nanodisks were observed by TEM. The nanodisks were composed of nanocrystallites with a size of around $5 \mathrm{~nm}$ (Fig. 3a). This was in accordance with dark-field TEM observations (Fig. S6a-c $\dagger$ ). The crystalline polymorph was identified as vaterite from its corresponding selected-area electron diffraction (SAED) pattern (Fig. 3b). This polymorph characterization was also supported by the X-ray diffraction (XRD) pattern (Fig. S7 $\dagger$ ) and Fourier transform infrared (FTIR) spectrum (Fig. S8 †) of the nanodisks. Despite their polycrystalline structure, an arched pattern was observed in the SAED analysis (Fig. 3b), indicating that the nanodisks had a preferred crystallographic orientation. The direction of the $c$ axis of vaterite was vertical to the in-plane direction of the vaterite nanodisks. We then obtained atomic-level structural information for the vaterite nanodisks. Fig. $3 \mathrm{c}$ shows a cryoHRTEM image of the part of a nanodisk indicated in a low-

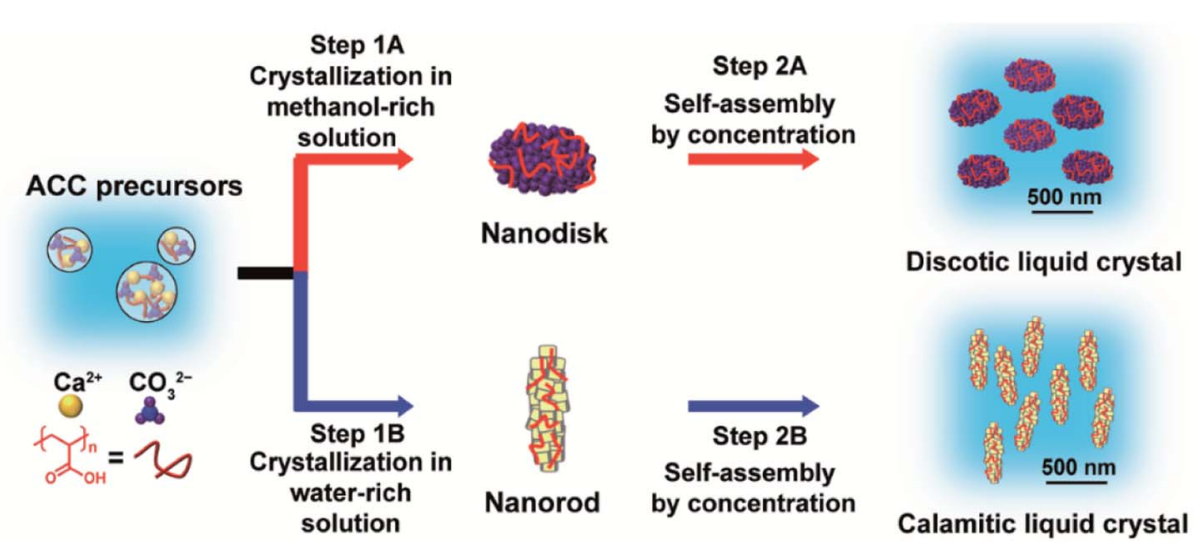

Fig. 1 Schematic illustration of biomineralization-inspired methods for selective synthesis of discotic and calamitic liquid crystals through crystallization control of poly(acrylic acid) (PAA)-stabilized amorphous calcium carbonate (ACC) precursors by changing the concentration ratio of methanol to water in the crystallization solution. The purple spheres in the nanodisk and the yellow tetrahedrons in the nanorod show vaterite and calcite nanocrystallites, respectively. The red curved lines represent PAA molecules. 
(a)

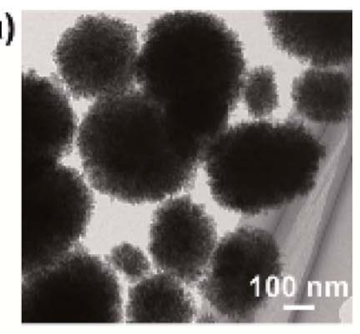

(b)
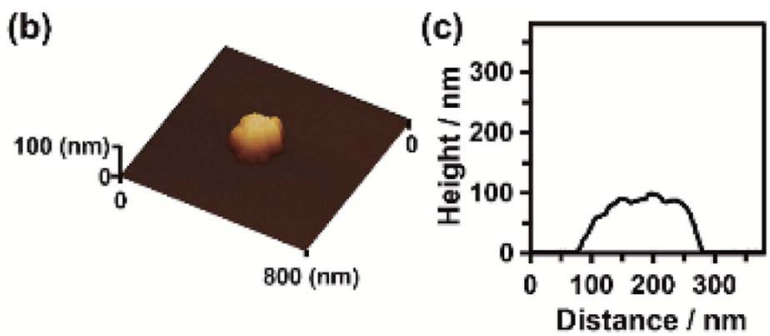

(d)
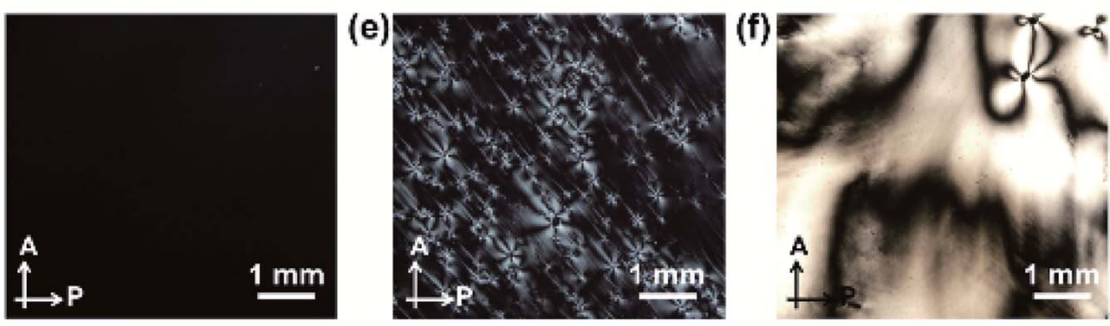

Fig. 2 (a) TEM image of $\mathrm{CaCO}_{3}$-based nanodisks obtained after crystallizing the ACC precursors in methanol/water (7: 3) solution in the presence of $25 \mathrm{mM} \mathrm{Na}_{2} \mathrm{CO}_{3}$. (b) AFM image of a $\mathrm{CaCO}_{3}$-based nanodisk on an Si substrate. (c) The height profile of the nanodisk. POM images of aqueous colloidal dispersions of $\mathrm{CaCO}_{3}$-based nanodisks with concentrations of (d) 1.7 vol\%, (e) 16 vol\%, and (f) 22 vol\%. A: analyzer. P: polarizer.

magnification TEM image (Fig. 3d). Crossed lattice fringes were clearly observed, which were assigned to vaterite crystals based on their fast Fourier transform (FFT) pattern (Fig. 3e). The crystallographic orientation was maintained between adjacent nanocrystallites. The vaterite disks were easily damaged at the atomic scale by electron irradiation at $120 \mathrm{kV}$ and room temperature, probably because vaterite is the most unstable of the $\mathrm{CaCO}_{3}$ crystalline polymorphs. We explored the use of cryoHRTEM with a cryo-TEM holder under low electron dose. This approach suppressed electron irradiation damage and enabled structural analyses of nanodisks with atomic resolution. In addition, atomic-level structural information was obtained using a negative spherical aberration imaging technique of HRTEM, which was optimized to display brighter contrast at atom positions. ${ }^{46,47}$ The oriented structure of nanocrystallites shown in Fig. 3c suggests that the nanodisks formed through oriented attachment of nanoparticles, which is widely observed in biomineralization and bioinspired crystallization. ${ }^{48,49}$ An amorphous layer with a thickness of around 1-2 $\mathrm{nm}$ was observed on the surfaces of the nanodisks. Thermogravimetric (TG) analysis revealed that the vaterite nanodisks were hybridized with $7.9 \mathrm{wt} \%$ PAA molecules (Fig. S9†) and the surfaces of the nanodisks were negatively charged (Fig. S5 $\dagger$ ). These results suggest that the amorphous layer consisted of PAA molecules. The vaterite nanodisks complexed with PAA molecules were stable in aqueous phase at least for one month.

TEM observation showed that the nanorods possessed polycrystalline structures composed of nanocrystallites with a size of around $20 \mathrm{~nm}$ (Fig. 3f). Dark-field TEM observation also showed the polycrystalline structure of the nanorods (Fig. S6d-fi†). Based on the SAED pattern, the polymorph was identified as calcite (Fig. 3g), which was consistent with the XRD pattern (Fig. S7†) and FTIR spectrum (Fig. S8 $\dagger$ ) of the nanorods. The polycrystals had a preferred orientation with the $c$ axis of calcite directed along the long axis of the calcite nanorods (Fig. 3g). Atomic-scale observations of the calcite nanorods were conducted using HRTEM at room temperature because of their higher stability against electron dose than that of the nanodisks. An HRTEM image (Fig. 3h) was collected in the region of a nanorod indicated in Fig. 3i. The observed lattice fringes corresponded to the (006) of calcite according to the FFT pattern (Fig. 3j). Connection of two adjacent nanocrystallites was also observed. The crystallographic orientations of these two nanocrystallites were the same, suggesting that the oriented attachment of nanoparticles was involved in the formation mechanism of the nanorods. ${ }^{48,49}$ The surfaces of the crystalline structures were covered with amorphous layers with thicknesses of around 1-2 nm. The amorphous pattern of the surface layer was probably observed more clearly for the nanorods compared with for the nanodisks because of the higher electron dose and lower signal-to-noise ratio for the observation of the former compared with that of the latter. As for the nanorods, the amorphous layers are considered to be composed of PAA molecules based on the results of TG analysis (Fig. S9†) and zeta potential measurements (Fig. S5†).

These atomic-scale structural analyses using state-of-the-art HRTEM techniques revealed that both of these different types of $\mathrm{LC}$ materials have similar $\mathrm{CaCO}_{3} / \mathrm{PAA}$ hybrid structures with preferred crystallographic orientations although they have different morphologies and polymorphs. The organic layer of PAA on the surface of these mesogenic nanoparticles provided the colloidal stability (Fig. S4 and S5†). It is presumed that the preferred crystallographic orientation of $\mathrm{CaCO}_{3}$ crystals within the nanocomposites led to the optical anisotropy observed by POM (Fig. 2f and S3d $\dagger$ ) because of the intrinsic birefringence of calcite $^{50}$ and vaterite crystals. ${ }^{51,52}$ Our findings indicate that biomineralization-inspired self-organization of $\mathrm{CaCO}_{3}$ and PAA is a promising approach for design of different types of organic/ inorganic hybrid colloidal liquid crystals. 
(a)

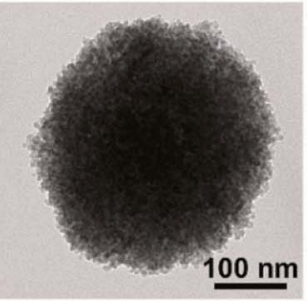

(b)

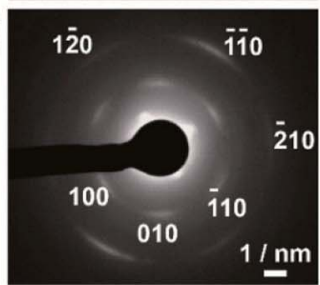

(f)

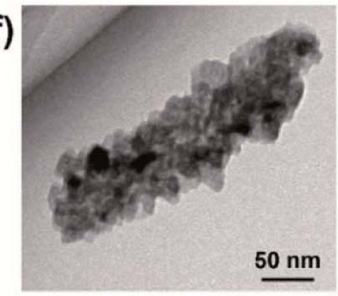

(g)

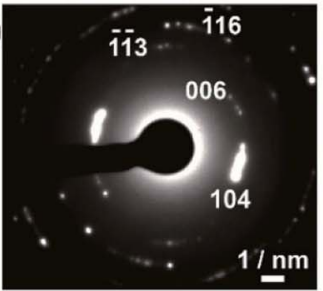

(c)

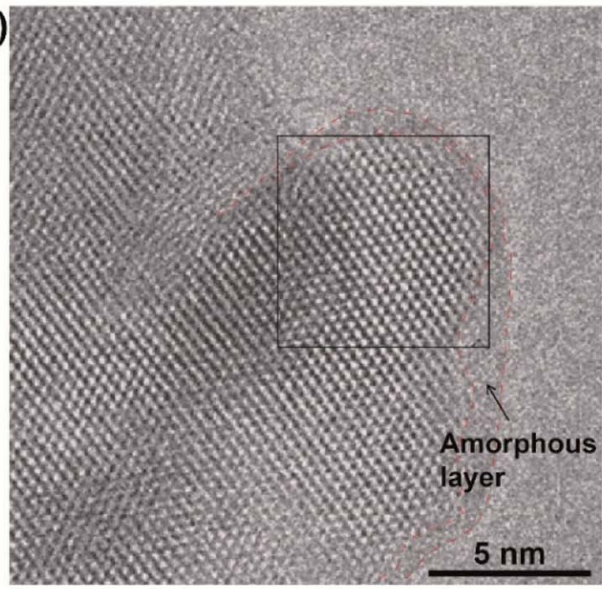

(h)

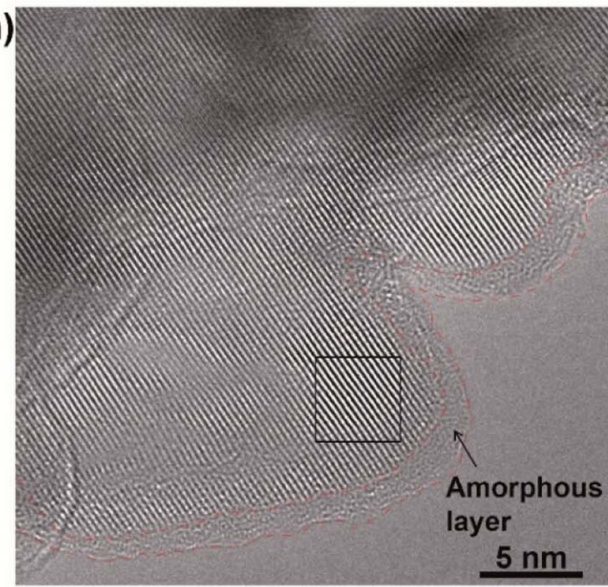

(d)

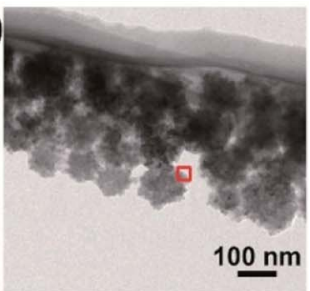

(e)

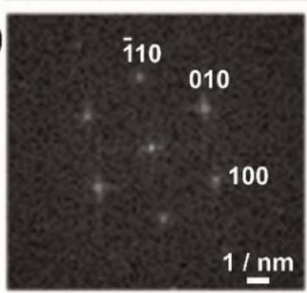

(i)

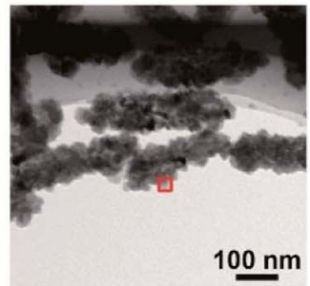

(j)

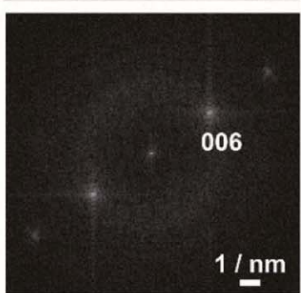

Fig. 3 (a) TEM image of a calcium carbonate $\left(\mathrm{CaCO}_{3}\right)$-based nanodisk. (b) Selected-area electron diffraction (SAED) pattern of the nanodisk. (c) Cryogenic high-resolution TEM (cryo-HRTEM) image of a $\mathrm{CaCO}_{3}$-based nanodisk corresponding to the red square in (d). (d) TEM image of $\mathrm{CaCO}_{3}$-based nanodisks at low magnification. (e) Fast Fourier transform (FFT) pattern corresponding to the black square in (c). (f) TEM image of a $\mathrm{CaCO}_{3}$-based nanorod. (g) SAED pattern of the nanorod. (h) HRTEM image of a $\mathrm{CaCO}_{3}$-based nanorod corresponding to the red square in (i). (i) TEM image of $\mathrm{CaCO}_{3}$-based nanorods at low magnification. (j) Fast FFT pattern corresponding to the black square in (h). Amorphous layers are surrounded by red dashed lines.

Two different types of LC materials, nanodisks and nanorods, were obtained from the same precursors. The precursors stabilized with PAA molecules were observed by TEM (Fig. S10a $\dagger$ ) and SEM (Fig. S11†). The precursors possessed spherical morphologies. Dynamic light scattering (DLS) measurements showed that the average diameter of the precursors was $23 \mathrm{~nm}$ (Fig. S10c $\dagger$ ). These results were in good agreement with TEM (Fig. S10a†) and SEM (Fig. S11†) observations. The polymorph of the nanoparticle precursors was characterized as ACC (Fig. S7, S8, and S10b $\dagger$ ). The surface potential of the precursor nanoparticles was $-12.7 \pm 5 \mathrm{mV}$ (Fig. S5 $\dagger$ ). According to TG analysis (Fig. S9†), the ACC precursors were composed of $66 \mathrm{wt} \% \mathrm{CaCO}_{3}, 20 \mathrm{wt} \% \mathrm{PAA}$, and $14 \mathrm{wt} \%$ $\mathrm{H}_{2} \mathrm{O}$. This suggests that the ACC nanoparticles were stabilized through hybridization with PAA.

Transformation of ACC into crystalline polymorphs is still far from being comprehensively understood. ${ }^{36-40}$ Likewise, the selective formation mechanisms for composite nanodisks and nanorods in the present study also remain unclear. However, we assume that these ACC precursors were transformed into
calcite/PAA hybrid nanorods and vaterite/PAA hybrid nanodisks through different crystallization pathways. During the formation process of nanorods over 3 days, the turbidity of the colloidal dispersions of ACC precursors disappeared and then recovered (Fig. S12†). This suggests that dissolution and reprecipitation processes were involved in the transformation of ACC precursors into nanorods. In contrast, such solution turbidity changes were not observed during the formation process of vaterite/PAA hybrid nanodisks. The crystallization rate of nanodisks was much slower than that of nanorods; it took one month to complete the transformation of ACC precursors into nanodisks. The solid-state amorphous-crystalline transition might be dominant in methanol/water mixtures because of the lower dissolution constant of ACC precursors in methanol than in water.

We explored the effects of the concentration ratios of methanol to water in the crystallization solutions on the polymorphs and morphologies of crystals obtained from the ACC precursors. When the volume ratio of methanol to water was below $30 \%$, the obtained crystals were calcite (Fig. 4a). Vaterite 
crystals coexisted with calcite crystals in the systems with methanol volume ratios of $40 \%$ to $60 \%$ (Fig. $4 \mathrm{a}$ and S13†). When the methanol concentration was increased to $70 \%$, vaterite crystals formed exclusively. These results based on XRD patterns were in good agreement with FTIR results (Fig. S13 $\dagger$ ). For the system with a methanol volume fraction of $80 \%$, crystallization of ACC was not detected even after one month. Further increasing the methanol fraction caused aggregation and sedimentation of ACC nanoparticle precursors. The crystals formed in the mixtures with various concentrations of methanol were observed by SEM. The SEM observations showed that nanorods formed when the methanol concentration was below 30\% (Fig. 4b-e), whereas both nanodisks and nanorods formed simultaneously at higher concentrations of methanol (Fig. 4f-h). When the methanol volume fraction was increased to $70 \%$, only nanodisks were observed (Fig. 4i). These results indicate that fine tuning of the solvent composition of crystallization solutions for ACC precursors is important for the selective synthesis of composite nanodisks and nanorods.

\section{Conclusions}

In conclusion, discotic colloidal liquid crystals composed of $\mathrm{CaCO}_{3}$-based nanodisk composites were developed. We achieved selective synthesis of LC nanodisk and nanorod composites by simply tuning the composition of the crystallization solution containing the PAA-stabilized ACC precursors- an approach inspired by biomineralization. Advanced TEM analyses clarified the atomic-scale $\mathrm{CaCO}_{3} / \mathrm{PAA}$ hybrid structures of the composites, showing that the self-organized hybrid structures were suitable for the formation of colloidal LC states. These $\mathrm{CaCO}_{3}$-based colloidal liquid crystals are promising as functional green and sustainable materials because of the biofriendliness of $\mathrm{CaCO}_{3}$, their anisotropic properties as LC materials, and the energy-efficient synthetic procedure. These $\mathrm{CaCO}_{3}$-based colloidal materials with well-controlled shapes and sizes would be useful as drug carriers for efficient cellular uptake. ${ }^{53}$ In addition, the present methods using amorphous inorganic precursors stabilized with organic molecules provide important insights to facilitate design of new colloidal liquid crystals.

\section{Experimental}

\section{Synthesis of colloidal dispersions of ACC precursors}

A $100 \mathrm{mM}$ aqueous $\mathrm{CaCl}_{2}$ solution containing $\operatorname{PAA}\left(M_{\mathrm{w}}=2.0 \times 10^{3}\right.$, $7.2 \times 10^{-1} \mathrm{wt} \%$ ) was prepared. A $100 \mathrm{mM}$ aqueous $\mathrm{Na}_{2} \mathrm{CO}_{3}$ solution was also prepared. Equal volumes of these two solutions were mixed. The mixed solution was stirred for $1 \mathrm{~h}$ at room temperature and then centrifuged at $10000 \mathrm{~g}$ for $5 \mathrm{~min}$. The supernatant was decanted. The sediment was redispersed in deionized water. The colloidal dispersion was centrifuged at $10000 \mathrm{~g}$ for $5 \mathrm{~min}$. The concentrated colloidal dispersion of ACC nanoparticles was

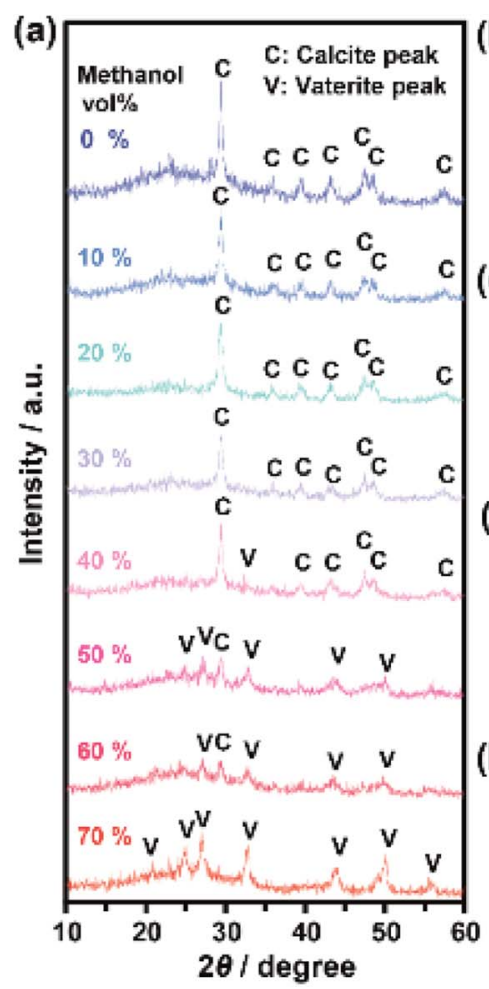

(b)

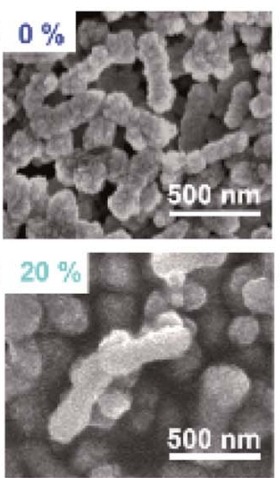

(c)

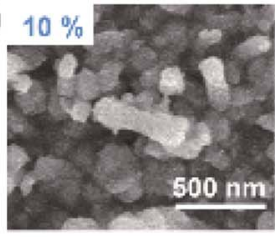

(e)

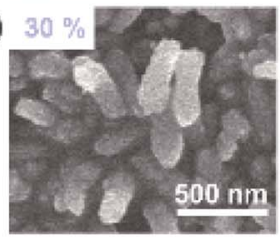

(f)

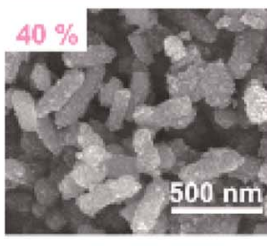

(g)

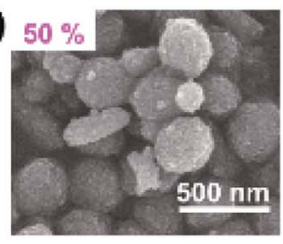

(h)

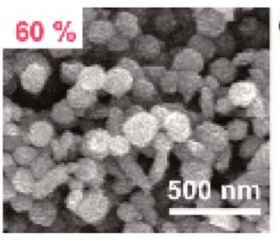

(i)

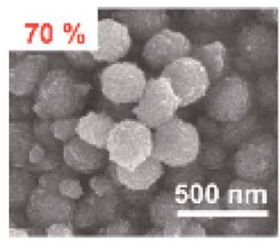

Fig. 4 (a) XRD patterns for $\mathrm{CaCO}_{3}$-based nanocrystals obtained by crystallization of the $\mathrm{ACC}$ precursors in $25 \mathrm{mM} \mathrm{Na}_{2} \mathrm{CO}_{3}$ aqueous solution containing methanol volume fractions of $0 \%, 10 \%, 20 \%, 30 \%, 40 \%, 50 \%, 60 \%$, and $70 \%$. SEM images of CaCO 3 -based nanocrystals obtained by crystallization of the ACC precursors in $25 \mathrm{mM} \mathrm{Na}_{2} \mathrm{CO}_{3}$ aqueous solution containing methanol volume fractions of (b) $0 \%$, (c) $10 \%$, (d) $20 \%$, (e) $30 \%$, (f) $40 \%$, (g) $50 \%$, (h) $60 \%$, and (i) $70 \%$. 
collected for use as precursors for syntheses of vaterite/PAA hybrid nanodisks and calcite/PAA hybrid nanorods before drying.

\section{Synthesis of colloidal dispersions of vaterite/PAA hybrid nanodisks}

The collected ACC precursors were dispersed in methanol/water (7:3 in the volume ratio) mixed solution containing $\mathrm{Na}_{2} \mathrm{CO}_{3}$ (25 mM). The dispersion of ACC precursors was stirred for 1 month at room temperature. The dispersion was centrifuged at $10000 \mathrm{~g}$ for $5 \mathrm{~min}$ and then the supernatant was removed. The sediment was redispersed in deionized water. The colloidal dispersion was centrifuged at $10000 \mathrm{~g}$ for $5 \mathrm{~min}$ to obtain the colloidal liquid crystal. Finally, deionized water was added to the liquid crystal to adjust the concentration.

\section{Synthesis of colloidal dispersions of calcite/PAA hybrid nanorods}

The collected ACC precursors were dispersed in $25 \mathrm{mM} \mathrm{Na} \mathrm{CO}_{3}$ aqueous solution. The dispersion was stirred for 3 days at room temperature. The dispersion was centrifuged at $10000 \mathrm{~g}$ for $5 \mathrm{~min}$ and then the supernatant was removed. The sediment was redispersed in deionized water. The colloidal dispersion was centrifuged at $10000 \mathrm{~g}$ for $5 \mathrm{~min}$ to obtain the colloidal liquid crystal. Finally, deionized water was added to the liquid crystal to adjust the concentration.

\section{Characterization}

XRD patterns were recorded using a SmartLab (Rigaku) diffractometer with $\mathrm{Cu} \mathrm{K} \alpha$ radiation. FTIR spectra were obtained with a JASCO/FTIR-660 Plus spectrometer using the $\mathrm{KBr}$ method. TG measurements (Rigaku, TG-8120) were performed up to $1000{ }^{\circ} \mathrm{C}$ at a heating rate of $10{ }^{\circ} \mathrm{C} \mathrm{min}{ }^{-1}$ under air flow $\left(100 \mathrm{~mL} \mathrm{~min}^{-1}\right)$. The optical properties were investigated using a polarizing optical microscope (Olympus, BX51) for colloidal dispersions of vaterite nanodisks and calcite nanorods at various concentrations. Crystal morphologies were examined using SEM (Hitachi, S-4700) with prior conductive treatment using a Hitachi E-1030 ion sputterer. TEM characterizations were performed using a JEM-2800 (JEOL) electron microscope operated at $100 \mathrm{kV}$. The samples for TEM observations were obtained by drying of diluted aqueous colloidal dispersions of ACC precursors, vaterite nanodisks and calcite nanorods placed on TEM grids. HRTEM images were obtained using an aberration-corrected TEM (JEOL, JEM-ARM200F) at $120 \mathrm{kV}$. To optimize a high-contrast structure image at low-electron-dose observation, we applied the negative spherical aberration imaging condition. ${ }^{47}$ Cryo-HRTEM was conducted using a cryoTEM holder (Gatan, 636 cooling holder) filled with liquid nitrogen. DLS and zeta potential measurements were performed using a Zetasizer (Nano-ZS, Malvern Instruments Ltd.) for diluted aqueous colloidal dispersions of ACC precursors, vaterite nanodisks and calcite nanorods. AFM observation was carried out using an L-trace II (SII NanoTechnology, Inc.) microscope. The samples for AFM observations were obtained by drying of spin-coated diluted aqueous colloidal dispersions of vaterite nanodisks on $\mathrm{Si}$ substrates.

\section{Materials}

All chemical reagents used to synthesize $\mathrm{CaCO}_{3}$ crystals were obtained from commercial sources. PAA $\left(M_{\mathrm{w}}=2.0 \times 10^{3}\right)$ was purchased from Polysciences, Inc. (Warrington, PA, USA). $\mathrm{CaCl}_{2}$ was obtained from Wako Pure Chemicals Industries, Ltd. (Osaka, Japan). $\mathrm{Na}_{2} \mathrm{CO}_{3}$ was purchased from Kanto Chemical (Tokyo, Japan). All reagents were used as received.

\section{Conflicts of interest}

There are no conflicts to declare.

\section{Acknowledgements}

This study was partly supported by the Japan Society for the Promotion of Science (JSPS) KAKENHI (Grant Number JP19H05715, JP15H02179 and JP17J09259). M. N. is grateful for financial support from a JSPS Research Fellowship for Young Scientists and the JSPS Program for Leading Graduate Schools (MERIT). We thank Dr Satoshi Yamaguchi for performing the zeta potential measurements. TEM and AFM observations were conducted at the Advanced Characterization Nanotechnology Platform at the University of Tokyo, which is supported by the "Nanotechnology Platform" of the Ministry of Education, Culture, Sports, Science and Technology (MEXT), Japan. We thank Natasha Lundin, PhD, from Edanz Group (http:// www.edanzediting.com/ac) for editing a draft of this manuscript.

\section{Notes and references}

1 Handbook of Liquid Crystals, ed. J. W. Goodby, P. J. Collings, T. Kato, C. Tschierske, H. Gleeson and P. Raynes, Wiley-VCH, Weinheim, 2nd edn, 2014.

2 T. Kato, J. Uchida, T. Ichikawa and T. Sakamoto, Angew. Chem., Int. Ed., 2018, 57, 4355-4371.

3 H. N. W. Lekkerkerker and G. J. Vroege, Philos. Trans. R. Soc., A, 2013, 371, 20120263.

4 P. Davidson, in Handbook of Liquid Crystals, ed. J. W. Goodby, P. J. Collings, T. Kato, C. Tscierske, H. Gleeson and P. Raynes, Wiley-VCH, Weinheim, 2nd edn, 2014, vol. 8, ch. 27, pp. 819-836.

5 J.-F. Revol and R. H. Marchessault, Int. J. Biol. Macromol., 1993, 15, 329-335.

6 J.-F. Revol, H. Bradford, J. Giasson, R. H. Marchessault and D. G. Gray, Int. J. Biol. Macromol., 1992, 14, 170-172.

7 M. Siavashpouri, C. H. Wachauf, M. J. Zakhary, F. Praetorius, H. Dietz and Z. Dogic, Nat. Mater., 2017, 16, 849-856.

8 W. J. Chung, J. W. Oh, K. Kwak, B. Y. Lee, J. Meyer, E. Wang, A. Hexemer and S. W. Lee, Nature, 2011, 478, 364-368.

9 S. W. Lee, C. B. Mao, C. E. Flynn and A. M. Belcher, Science, 2002, 296, 892-895.

10 T. Sawada, Y. Murata, H. Marubayashi, S. Nojima, J. Morikawa and T. Serizawa, Sci. Rep., 2018, 8, 5412.

11 J. Kim, S. Michelin, M. Hilbers, L. Martinelli, E. Chaudan, G. Amselem, E. Fradet, J. P. Boilot, A. M. Brouwer, 
C. N. Baroud, J. Peretti and T. Gacoin, Nat. Nanotechnol., 2017, 12, 914-919.

12 E. Paineau, M. E. M. Krapf, M. S. Amara, N. V. Matskova, I. Dozov, S. Rouzière, A. Thill, P. Launois and P. Davidson, Nat. Commun., 2016, 7, 10271.

13 N. Miyamoto and T. Nakato, Isr. J. Chem., 2012, 52, 881-894.

14 M. Nakayama, S. Kajiyama, T. Nishimura and T. Kato, Chem. Sci., 2015, 6, 6230-6234.

15 M. Nakayama, S. Kajiyama, A. Kumamoto, T. Nishimura, Y. Ikuhara, M. Yamato and T. Kato, Nat. Commun., 2018, 9, 568.

16 T. Hoshino, M. Nakayama, S. Fujinami, T. Nakatani, Y. Kohmura and T. Kato, Soft Matter, 2019, 15, 3315-3322.

17 M. Nakayama, W. Q. Lim, S. Kajiyama, A. Kumamoto, Y. Ikuhara, T. Kato and Y. Zhao, ACS Appl. Mater. Interfaces, 2019, 11, 17759-17765.

18 H. B. Yao, J. Ge, L. B. Mao, Y. X. Yan and S. H. Yu, Adv. Mater., 2014, 26, 163-188.

19 A. Arakaki, K. Shimizu, M. Oda, T. Sakamoto, T. Nishimura and T. Kato, Org. Biomol. Chem., 2015, 13, 974-989.

20 B. Cantaert, D. Kuo, S. Matsumura, T. Nishimura, T. Sakamoto and T. Kato, ChemPlusChem, 2017, 82, 107-120.

21 F. Nudelman and N. A. J. M. Sommerdijk, Angew. Chem., Int. Ed., 2012, 51, 6582-6596.

22 U. G. K. Wegst, H. Bai, E. Saiz, A. P. Tomsia and R. O. Ritchie, Nat. Mater., 2015, 14, 23-36.

23 M. Suzuki, K. Saruwatari, T. Kogure, Y. Yamamoto, T. Nishimura, T. Kato and H. Nagasawa, Science, 2009, 325, 1388-1390.

24 L. K. Grunenfelder, S. Herrera and D. Kisailus, Small, 2014, 10, 3207-3232.

25 Handbook of Biomineralization, ed. E. Baeuerlein, P. Behrens and M. Epple, Wiley-VCH, Weinheim, 2007.

26 T. Mass, A. J. Giuffre, C.-Y. Sun, C. A. Stifler, M. J. Frazier, M. Neder, N. Tamura, C. V. Stan, M. A. Marcus and P. U. P. A. Gilbert, Proc. Natl. Acad. Sci. U. S. A., 2017, 114, E7670-E7678.

27 A. Gal, K. Kahil, N. Vidavsky, R. T. Devol, P. U. P. A. Gilbert, P. Fratzl, S. Weiner and L. Addadi, Adv. Funct. Mater., 2014, 24, 5420-5426.

28 S. Weiner and L. Addadi, Annu. Rev. Mater. Res., 2011, 41, 2140.

29 R. T. Devol, C. Y. Sun, M. A. Marcus, S. N. Coppersmith, S. C. B. Myneni and P. U. P. A. Gilbert, J. Am. Chem. Soc., 2015, 137, 13325-13333.

30 S. Kamat, X. Su, R. Ballarini and A. H. Heuer, Nature, 2000, 405, 1036-1040.

31 M. Suzuki, T. Kogure, S. Weiner and L. Addadi, Cryst. Growth Des., 2011, 11, 4850-4859.

32 C. E. Killian, R. A. Metzler, Y. U. T. Gong, I. C. Olson, J. Aizenberg, Y. Politi, F. H. Wilt, A. Scholl, A. Young, A. Doran, M. Kunz, N. Tamura, S. N. Coppersmith and P. U. P. A. Gilbert, J. Am. Chem. Soc., 2009, 131, 18404-18409.
33 Y. Ma, B. Aichmayer, O. Paris, P. Fratzl, A. Meibom, R. A. Metzler, Y. Politi, L. Addadi, P. U. P. A. Gilbert and S. Weiner, Proc. Natl. Acad. Sci. U. S. A., 2009, 106, 6048-6053. 34 A. G. Checa, A. B. Rodriguez-Navarro and F. J. EstebanDelgado, Biomaterials, 2005, 26, 6404-6414.

35 A. G. Checa, J. H. E. Cartwright and M.-G. Willinger, Proc. Natl. Acad. Sci. U. S. A., 2009, 106, 38-43.

36 J. J. De Yoreo, P. U. P. A. Gilbert, N. A. J. M. Sommerdijk, R. L. Penn, S. Whitelam, D. Joester, H. Zhang, J. D. Rimer, A. Navrotsky, J. F. Banfield, A. F. Wallace, F. M. Michel, F. C. Meldrum, H. Cölfen and P. M. Dove, Science, 2015, 349, aaa6760.

37 M. H. Nielsen, S. Aloni and J. J. De Yoreo, Science, 2014, 345, 1158-1162.

38 P. J. M. Smeets, K. R. Cho, R. G. E. Kempen, N. A. J. M. Sommerdijk and J. J. De Yoreo, Nat. Mater., 2015, 14, 394-399.

39 Y. Xu, K. C. H. Tijssen, P. H. H. Bomans, A. Akiva, H. Friedrich, A. P. M. Kentgens and N. A. J. M. Sommerdijk, Nat. Commun., 2018, 9, 2582.

40 E. M. Pouget, P. H. H. Bomans, J. A. C. M. Goos, P. M. Frederik, G. De With and N. A. J. M. Sommerdijk, Science, 2009, 323, 1455-1458.

41 B. V. Parakhonskiy, A. M. Yashchenok, S. Donatan, D. V. Volodkin, F. Tessarolo, R. Antolini, H. Mçhwald and A. G. Skirtach, ChemPhysChem, 2014, 15, 2817-2822.

42 A. Yashchenok, B. Parakhonskiy, S. Donatan, D. Kohler, A. Skirtachade and H. Mohwald, J. Mater. Chem. B, 2013, 1, 1223-1228.

43 J. Yu, M. Lei, B. Cheng and X. Zhao, J. Solid State Chem., 2004, 177, 681-689.

44 Y. Hui, L. Tong, T. Weijun and G. Changyou, Chem. J. Chin. Univ., 2018, 39, 172-177.

45 Y. Oaki, S. Kajiayama, T. Nishimura, H. Imai and T. Kato, Adv. Mater., 2008, 20, 3633-3637.

46 C. L. Jia, L. Houben, A. Thust and J. Barthel, Ultramicroscopy, 2010, 110, 500-505.

47 M. Lentzen, Microsc. Microanal., 2008, 14, 16-26.

48 E. V. Sturm and H. Cölfen, Chem. Soc. Rev., 2016, 45, 58215833.

49 R. Q. Song and H. Cölfen, Adv. Mater., 2010, 22, 1301-1330. 50 S. Hirano, T. Yogo and K. Kikuta, Prog. Cryst. Growth Charact., 1991, 23, 341-367.

51 J. Johnston, H. E. Merwin and E. D. Williamson, Am. J. Sci., 1916, 41, 473-512.

52 S. J. Parkin, R. Vogel, M. Persson, M. Funk, V. L. Y. Loke, T. A. Nieminen, N. R. Heckenberg and H. RubinszteinDunlop, Opt. Express, 2009, 17, 21944-21955.

53 B. Parakhonskiy, M. V. Zyuzin, A. Yashchenok, S. CarregalRomero, J. Rejman, H. Möhwald, W. J. Parak and A. G. Skirtach, J. Nanobiotechnol., 2015, 13, 53. 